\title{
Children with speech difficulties: An exploratory survey of clinical practice in the Western Cape
}

\author{
Michelle Pascoe \\ Zinhle Maphalala \\ Aeysha Ebrahim \\ Daneil Hime \\ Bathobile Mdladla \\ Nerosha Mohamed \\ Mandy Skinner \\ Division of Communication Sciences and Disorders, Faculty of Health Sciences, University of Cape Town
}

Correspondence to: M Pascoe (michelle.pascoe@uct.ac.za)

\section{ABSTRACT}

This study investigated the methods of assessment and intervention used by speech-language therapists (SLTs) in the Western Cape when working with children with speech difficulties. Children with speech difficulties are likely to form a considerable part of SLT caseloads in South Africa, but assessment choice may not be clear-cut given the linguistic diversity of the region and that few assessments have been developed specifically for the SA population. Selection of intervention approaches may also pose difficulties, linked to the lack of assessments and the limited evidence base in our context. A questionnaire was sent to SLTs working with pre- and/ or primary-school-aged children. Twenty-nine clinicians responded (18.7\% response rate). The majority (89\%) use informal assessment in combination with formal assessment. When using formal assessments, more than $50 \%$ of SLTs surveyed make procedural or linguistic modifications. Participants used a variety of interventions such as auditory discrimination and phonological awareness, often in combination, and based on a child's profile of difficulties. Forty-six per cent of SLTs felt unsure about the selection of assessments and intervention for bi/multilingual children. Clinical implications arising from this preliminary investigation are discussed together with some suggestions for developing knowledge of children's speech difficulties in South Africa.
\end{abstract}

Keywords: articulation, assessment, clinical practice, intervention, phonology, speech difficulties

Children with speech difficulties typically constitute a large proportion of speech language therapists' (SLTs') caseloads. While the prevalence of speech difficulties in South Africa is not yet known, prevalence estimates from the USA show that approximately $7.5 \%$ of children between 3 and 11 years of age experience speech difficulties that are clinically significant (Ruscello, 2008). In the UK, approximately 48000 children are referred with speech difficulties each year (Broomfield \& Dodd, 2004); these speech difficulties are described as being of a primary nature, not secondary to a known aetiology such as hearing impairment or cleft palate.

The term 'speech difficulties' refers to any difficulty that affects the speech processing system and results in reduced intelligibility (Pascoe, Stackhouse \& Wells, 2006). The population of children with speech difficulties is a heterogeneous one. Some children experience peripheral speech problems, e.g. articulation or structural-based difficulties; some have difficulties with auditory input processing (e.g. auditory discrimination), and others with stored phonological representations or a combination of these (Stackhouse \& Wells, 1997). In addition to the nature of the difficulties, the degree of difficulty may vary from mild, affecting a small subset of speech sounds, to more widespread involvement of the phonological/linguistic system (Dodd, 2005). In the present study, the term 'speech difficulties' is used to include all difficulties of speech processing and production of a primary nature (following Dodd, 2005; Stackhouse \& Wells, 1997).

A range of perspectives are adopted by SLTs to deal with the variation of the population: psycholinguistic, linguistic and medical approaches. The psycholinguistic approach attempts to understand a child's difficulties by examining their strengths and weaknesses, as well as pinpointing the underlying breakdown (Stackhouse \& Wells, 1997). For example, Stackhouse, Pascoe and Gardner (2006) investigated a child's speech and literacy development by focusing on speech input, output and phonological representations. These underlying speech processing skills are necessary not only for speech development but also for phonological awareness, the bridge that links speech and literacy development in hearing populations. A breakdown at any one of these levels will not only result in spoken language difficulties but may also lead to written language difficulties (Stackhouse \& Wells, 1997).

The linguistic approach categorises children in terms of their observed speech behaviours, e.g. children with phonological processes may be observed to use 'fronting' and 'stopping' irrespective of the reason causing the process or particular features affected (Grunwell, 1985). The linguistic approach can be useful as it may give detailed information regarding the child's phonological system and speech output that should be targeted to achieve more intelligible speech. Dodd (2005) describes a differential diagnostic approach to understanding speech sound difficulties whereby all children are classified into one of five categories based on the observed nature of their difficulties: phonological disorder (inconsistent); phonological disorder (consistent); phonological delay; articulation difficulty; or childhood apraxia of speech (CAS).

In contrast, the medical approach to speech difficulties centres around specific medical diagnoses and the typically observed cluster of signs associated with particular disorders (Stackhouse \& Wells, 1997). This approach does not focus on the individual strengths and weaknesses of a child, or on the observed patterns of speech and language use, but rather aims to group children who present with similar and fairly broadly defined difficulties (e.g. inconsistent speech, prosodic difficulties, general clumsiness) under one label. The advantage of the medical approach is that diagnostic labelling facilitates communication between professionals and can be helpful in managing causal factors, e.g. hearing loss (Stackhouse \& Wells, 1997). However, being able to fit a child into a specific group does not always equate to a ready-made therapy plan and children diagnosed with similar speech difficulties (e.g. CAS) are very likely to require different therapy approaches. The literature shows that children with similar speech difficulties can have different underlying problems, and it can therefore be a challenge for SLTs to select an appropriate approach based on available evidence. Clearly the frameworks that a therapist uses to understand speech difficulties will be reflected in his/her choice of assessment and therapy methods. 
The identification and management of speech difficulties in children is important because such difficulties can result in communication problems for the child. In addition, speech difficulties may place children at increased risk of experiencing psychosocial difficulties such as being ignored by peers or bullied (Nash, Stengelhofen \& Brown, 2002). A large body of evidence suggests that speech disorders may affect preliteracy and literacy skills (Reilly, Douglas \& Oates, 2004). Bird, Bishop and Freeman (1995) conducted a study which indicated that children with speech problems had difficulty with phonological awareness and experienced significant literacy problems, most notably with spelling, when compared with typically developing children of the same chronological age without any speech difficulties. Children who have not been treated by the time they begin formal schooling, roughly at a chronological age of 6 years, can be at greater risk of developing literacy difficulties which can in turn result in poor academic achievement. The type of speech difficulties that have been linked to such negative outcomes are thought to be phonologically based difficulties rather than articulation difficulties.

Joffe and Pring (2008) carried out a survey of 98 SLTs working with children with phonological difficulties in the UK. While the title of the study describes 'phonological problems', their questionnaire included questions about both articulation and phonology as well as CAS. Joffe and Pring aimed to move beyond anecdotal evidence about how SLTs manage such children and describe the assessments and interventions carried out in that context. British SLTs favoured the South Tyneside assessment of phonology (STAP) (Armstrong \& Ainley, 1988), an easily administered screening tool to assess children's speech, but faced difficulties in distinguishing between phonological disorder and CAS. Most SLTs used a combination of interventions, with minimal-pair therapy, auditory discrimination and phonological awareness therapy being particularly popular (Joffe \& Pring, 2008).

The literature on children's speech difficulties strongly suggests that children with speech disorders benefit from appropriately administered intervention (Bowen, 2009; Reilly et al., 2004), and there are a range of effective treatment methods available for facilitating a change in children's sound systems (see Williams, McLeod \& McCauley, 2010 for a review). In terms of phonology, these include minimal-pair approaches (e.g. Gierut, 1990; Weiner, 1981); core vocabulary (e.g. Dodd, 2005) and phonological awareness approaches (e.g. Gillon, 2000), which have been shown to be effective. Therapy aimed at addressing articulation difficulties is also purported to have positive outcomes (Bowen, 2009), while interventions for motor-speech difficulties such as CAS and dysarthria have had more variable outcomes (see Williams et al., 2010). The great majority of intervention studies focusing on children's speech have been carried out in developed nations; to date there is a paucity of information about the effectiveness of intervention for children with speech difficulties in the South African context.

\section{Speech difficulties in South African children}

South Africa is a country rich in linguistic diversity, with eleven official languages. In the Western Cape, the main languages spoken are Afrikaans, isiXhosa and English. Relatively little is known about the acquisition of South African English or Afrikaans phonology, and even less about isiXhosa. While there is a substantial amount of research surrounding children's speech sound acquisition in English (e.g. Grunwell, 1985; Dodd, 2005), most of this has been conducted with children in Europe, North America or Australia. Gxilishe (2004) conducted a study in the Western Cape, looking at the acquisition of clicks by Xhosa-speaking children. He found that at the onset of speech (approximately 1 year of age) Xhosa-speaking children begin using three basic clicks. Such studies are important in advancing our knowledge of speech and language development in the local context; however, further research is needed.

In South Africa monolingualism is rare. Most children presenting with speech difficulties will be bilingual or multilingual. This results in significant challenges in the provision of assessment and intervention services, especially as the vast majority of South African SLTs are currently English- or Afrikaans-speaking. Goldstein and Fabiano (2006) state that the difficulty in treating these children is caused by the lack of understanding of theories of bilingual phonological representation and the lack of knowledge of current best practices related to the assessment of and intervention for bi- and multilingual children. Children with speech difficulties are likely to form a considerable part of SLT caseloads in South Africa - if prevalence estimates from other countries are anything to go by (Broomfield \& Dodd, 2004; Ruscello, 2008) - but the choice of assessments may not be straightforward, given the linguistic diversity of the region and the fact that few assessments have been developed specifically for the SA population. Carter, Lees, Murira, Gona, Neville and Newton (2004) emphasise the need to develop culturally appropriate materials because of cultural variation and potential cultural bias. In their Kenyan-based study they found that very few clinicians assess or treat children from a culture different to their own. The vast majority of SLTs working in South Africa today are English- or Afrikaans-speaking whites, and as a result speakers of the indigenous languages (e.g. isiXhosa and isiZulu) have typically been under-served.

This study reports an exploratory survey of SLTs in the Western Cape working with children with speech difficulties. Current knowledge of clinical practice in the Western Cape, (as in the UK, Joffe \& Pring, 2008) is largely anecdotal and therefore the study aimed to find more reliable evidence about the assessment and therapies currently being used. SLTs' current experiences of working with children with speech difficulties were investigated with a view to using this information as a starting point to understanding the needs of clinicians in the region in terms of the knowledge needed, and assessment and intervention materials required.

\section{Methodology \\ Aim}

The aim was to investigate the clinical practice of SLTs in the Western Cape working with children with speech difficulties.

\section{Objectives}

1. To contextualise the general nature of the clinical practice of SLTs in the Western Cape working with children with speech difficulties.

2. To describe the assessment methods used by SLTs in the Western Cape working with children with speech difficulties.

3. To describe the intervention approaches used by SLTs in the Western Cape working with children with speech difficulties.

\section{Research design}

A descriptive, questionnaire-based survey design was used. A questionnaire was sent to SLTs working with pre- and/or primary-schoolaged children to investigate their experiences of working with children with speech difficulties. Survey research is commonly used as a means of collecting information about certain characteristics and practices in order to use it for descriptive purposes and has been widely used to study professional and clinical issues in the field of communication disorders (Maxwell \& Satake, 2006). The study was exploratory: it was used as a starting point to investigate issues relating to speech development and difficulties in a multilingual context. Exploratory research is typically used when research is in a preliminary stage and definitive conclusions arising from it are rare (Maxwell \& Satake, 2006).

\section{Participants}

\section{Selection criteria}

Participants were SLTs registered with the Health Professions Council of South Africa (HPCSA). These SLTs had to be currently working in the Western Cape with pre- and/or primary-school-aged children. The total population was estimated at 155 . Researchers attempted to gather data from the entire population. Those who responded to the questionnaire formed the sample for this study. Such convenience sampling targets individuals who are readily available to form a participant group and is often used for exploratory research (Maxwell \& Satake, 2006). 


\section{Description of materials}

The questionnaire used (Appendix A) was based on that of Joffe and Pring (2008), but adapted for the purpose of this study in two ways: (i) the conceptualisation of speech difficulties followed the psycholinguistic perspective of Stackhouse and Wells (1997) rather than being limited only to phonological difficulties; and (ii) the developing nature of the South African context and the linguistic diversity of the region was explored as it relates to children with speech difficulties.

The questionnaire consisted of: (i) open-ended questions, where the participants were encouraged to provide their own responses; and (ii) closed questions, where the participants were required to choose an option. The three objectives covered included: $(i)$ general questions about clinical practice; (ii) questions related to the methods of assessment; and (iii) questions related to the methods of intervention used by SLTs in the Western Cape. In section A participants were asked about their clinical experience and work setting, their own languages and those of their clients. In section B they were asked about the assessments used, including languages of assessment, challenges associated with assessment and theoretical frameworks that may help identify, assess and diagnose speech difficulties. Section $\mathrm{C}$ focused firstly on intervention for children with speech difficulties, and then, following Joffe and Pring (2008), listed questions about specific therapies for speech difficulties that have been documented in the literature. Questions were also included in this section regarding knowledge of the evidence base related to intervention for speech difficulties, and challenges in the South African context.

\section{Pilot}

The questionnaire was given to one SLT for completion, prior to the final mailing. Although a larger pilot sample would have been preferable, one pilot participant was all that was practical in a short time frame and from a relatively small population in this preliminary study. This SLT was asked to provide feedback on the covering letter, length of time taken to complete the questionnaire and the clarity of the questions. A few questions were reworded for improved clarity. The length of the questionnaire was acceptable and was therefore not adjusted.

\section{Procedures}

SLTs currently registered with the HPCSA and practising in the Western Cape were identified via mailing lists and databases from special interest groups, the South African Speech Language and Hearing Association (SASLHA), private practice organisations and through personal contacts. The questionnaire was sent to all SLTs via electronic mail and by post on request. One hundred and fifty-five questionnaires were sent out to SLTs in the Western Cape. Participants were given 2 months to respond to the questionnaire and electronic reminders were sent 3 weeks after the mailing of the first questionnaire.

\section{Ethical considerations}

The study received ethical approval from the University of Cape Town Research Ethics Committee. A covering letter containing information about the purpose of the study was included with the questionnaire. All SLTs who read the letter and sent back the completed questionnaire within the given timeframe were assumed to have given informed consent to participate in the study.

\section{Analysis}

When completed questionnaires were received, identifying information was removed and a numerical code was assigned in order to ensure anonymity. Data were entered into a Microsoft Excel spreadsheet. The first step of analysis was calculating the response rate, carried out as suggested by Maxwell and Satake (2006). The data were then quantitatively analysed using descriptive statistical analysis, and qualitatively analysed by summarising key themes/trends.

\section{Results}

One hundred and fifty-five questionnaires were sent out to SLTs in the Western Cape. Twenty-nine completed questionnaires (18.7\%) were returned. This is a low response rate, and the results should be interpreted with caution because respondents may be fundamentally different (i.e. more interested in research, more willing to engage with issues of linguistic diversity) from non-respondents. The low response rate may also suggest that many of the SLTs in the region are not in fact working directly with the population of children with speech difficulties; the nature of SLT work in a developing context may mean that speech difficulties are not regarded as a priority. Nevertheless, despite the low response rate, the data were considered important given the exploratory nature of the study. Of the 29 questionnaires received, 28 were included $(96.5 \%)$. One participant did not meet the criteria as $\mathrm{s} /$ he did not work with children.

\section{General nature of clinical practice}

Participants were divided into three groups: inexperienced therapists (from 1 to 3 years' working experience; $n=6 ; 22 \%$ ), experienced therapists ( 4 - 10 years' working experience; $n=9 ; 32 \%$ ), and very experienced therapists (more than 10 years' working experience; $n=13$; 46\%). Participants practise in a variety of settings: 8 (29\%) work in independent practice, 5 (18\%) work in government hospitals, 4 (14\%) work in government schools and 1 works in an independent school. The remainder of the participants $(n=10 ; 36 \%)$ work in a combination of these settings, as well as universities, private hospitals and nongovernmental organisations. The majority of participants $(n=19,68 \%)$ speak English and Afrikaans; 5 participants (18\%) speak English only; 2 participants (7\%) speak English, Afrikaans and German; 1 participant (4\%) speaks English, Afrikaans and isiXhosa; and 1 participant (4\%) speaks English and German. Figure 1 summarises these language data.

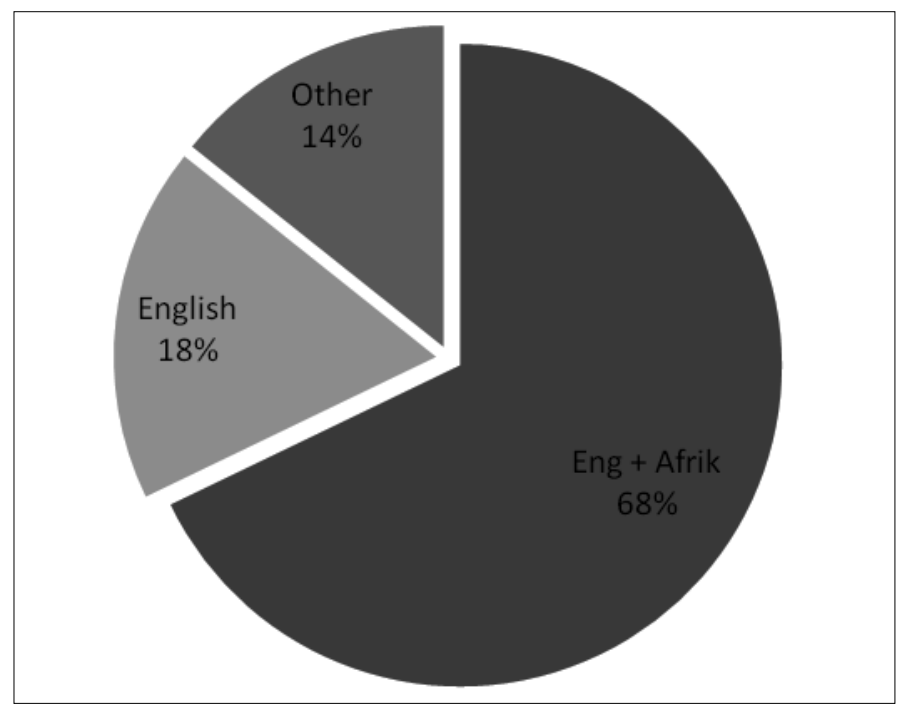

Fig. 1. Languages spoken by participants.

The majority $(54 \%, n=15)$ work with multilingual children who speak English, Afrikaans and isiXhosa; $21 \%(n=6)$ work with English-speaking children only and $18 \%(n=5)$ work with children who speak English and Afrikaans. Twenty-eight per cent of participants $(n=9)$ reported that they would always assess and give therapy using a child's home language, but many of these SLTs who emphasised home language were therapists who were either only working with monolingual children, or were bilingual English/Afrikaans speakers themselves who would then offer treatment in either language, depending on the home language of the child. Focusing on the 15 respondents who work with multilingual (English, Afrikaans and isiXhosa) children, only 6 (40\%) said that they would always assess and treat in the child's home language - whether it was English, Afrikaans or isiXhosa. One of these SLTs was able to speak isiXhosa, and the other 5 mentioned that they would work through interpreters, parents or colleagues. The majority $(60 \%, n=9)$ of those SLTs working with multilingual children will assess and manage those children in English (or in some cases English or Afrikaans) only. For the majority of the participants $(36 \% ; n=10)$ between $40 \%$ and $70 \%$ of the children they see have speech difficulties, a substantial proportion of their caseloads. 
Twenty-five per cent $(n=7)$ of the participants rated themselves very confident or confident about selection of assessments and providing therapy to a child who speaks another language to their own; $29 \%(n=8)$ rated themselves as neutral in this regard; $46 \%(n=13)$ rated themselves as not confident/not confident at all. Figure 2 shows these data.

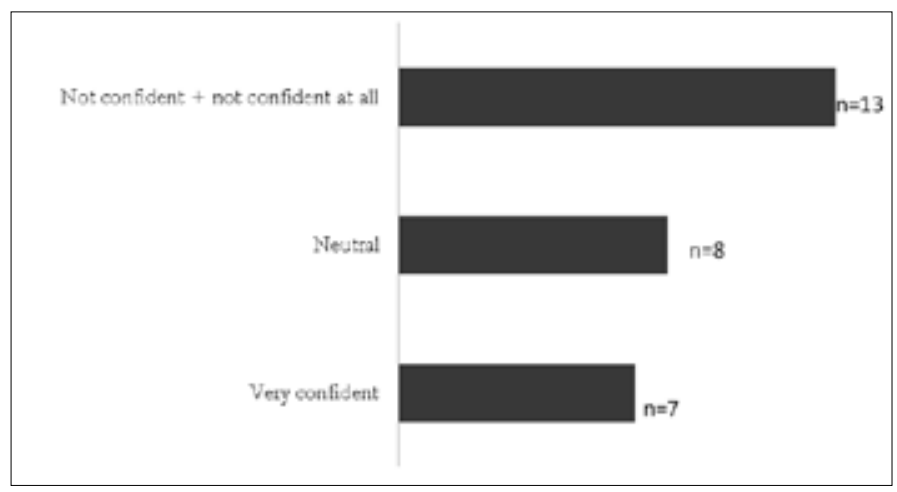

Fig. 2. Confidence levels of therapists when selecting assessments and providing therapy to bi/multilingual children with speech difficulties.

Among the participants who rated themselves confident or very confident, no specific pattern was identified in relation to their level of experience. However, it was found that of the 13 participants that rated themselves not very confident or not confident at all, 10 were very experienced (having practised for more than 10 years).

\section{Assessment}

Ten different formal assessments are used to assess children's speech. The Goldman-Fristoe articulation test (GFTA) (Goldman \& Fristoe, 1986 ) was the most popular with $32 \%(n=9)$ using it. Other assessments that were commonly used include: the assessment component of the Nuffield Centre Dyspraxia Programme (Williams \& Stephens, 2004) used by $14 \%(n=4)$ of the therapists, and the Edinburgh articulation test (Anthony, Bogle, Ingram, \& McIsaac, 1971) used by $10 \%(n=3)$ of the therapists. Other assessments mentioned included the phonological awareness test (Robertson \& Salter, 1995) and the test of auditory perceptual skills (Gardner, 1996). These results are shown in Figure 3. All of the assessments have been standardised on, or have been developed for use with, English-speaking monolingual populations, mainly in the UK and North America.

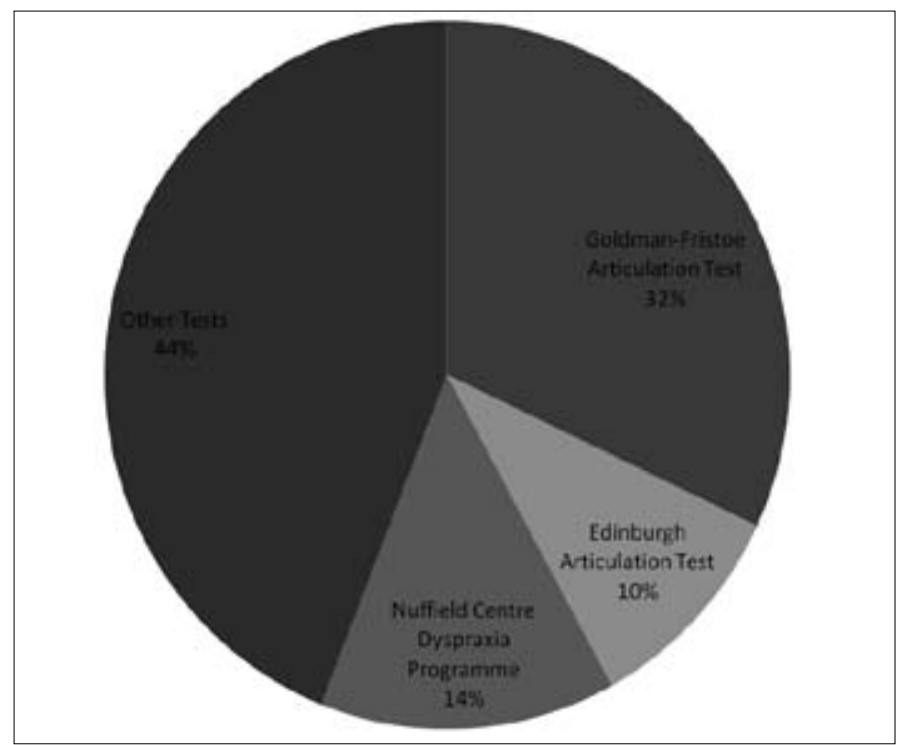

Fig. 3. Formal assessments of children's speech used by participants.

Eighty-nine per cent $(n=25)$ use informal assessment tools in addition to the formal assessments described. More than $50 \%$ of SLTs $(n=14)$ indicated that they make adaptations to formal tests to better suit the South African population, e.g. translating the assessment into another language and using more culturally appropriate material (e.g. pictures and objects). For example, one SLT reported that 'I have included "taxi" in my ... assessment - more culturally appropriate than "underground".' It was also noted that SLTs often change the administration method of tests; they often repeat instructions to encourage the child to respond: 'I explain the test differently or leave out sections.' Many of the standardised tests were being used as a set of picture stimuli to elicit speech, which was then qualitatively analysed and interpreted rather than following the prescribed scoring and analysis procedures. The same set of pictures was sometimes used to elicit words in a variety of languages.

Many of the SLTs $(n=10 ; 36 \%)$ elicit connected speech by story-telling and have made their own assessments. Typically these were described as sets of pictures that contain all the consonants of a given language, mostly for English and Afrikaans, although in a small number of cases $(n=2)$ participants mentioned a set of screening pictures for eliciting some isiXhosa speech sounds. One of the participants gave some detail of the materials used in her own test: ... 63 flash cards, targeting speech sounds [of English ]... Pictures are stickers of real items in colour and are of objects and items they should be familiar with.' Another participant stated 'I use picture cards, objects, play, history from parents, etc.' One of the participants attached a speech assessment s/he developed while an undergraduate student. The assessment contained pictures that could be used to elicit all the speech sounds of the English language and with pictures specifically selected for the South African context. The therapists who indicated that they had made their own speech tests all speak more than one language and treat children who speak more than one language themselves, but materials were developed mostly for English and Afrikaans speakers, and very few for isiXhosa speakers.

Participants were asked whether they would find an assessment useful if it could be used in all three predominant languages of the region. The majority of the participants, $(72 \%, n=20)$ agreed that they would find it helpful. The remaining participants $(29 \%, \mathrm{n} 8)$ said they would not find such an assessment useful for various reasons (their caseloads only comprised English-speaking children or they only felt confident in assessing and treating children in English).

Participants were asked to specify which additional materials they would find helpful when assessing a child's speech. These included accepted variations of speech sound production in the Western Cape and South Africa as well as standard norms, clinical judgement frameworks to assist in the judgement of disordered or delayed profiles, modern colourful pictures, pictures and objects for special populations, photograph-based tests with matching objects and a list of phonological processes in more languages.

The SLTs were asked about their frameworks or approaches to understanding children with speech difficulties. The majority $(68 \%$; $n=19$ ) use developmental approaches together with a diagnostic approach to understanding children with speech difficulties, e.g. ... show the parent the chart of norms for sound development ... i.e. a developmentally based target selection'; '[group in terms of] ... articulation or phonological delay. Sometimes say dyspraxia when oral motor planning difficulties are evident'; '[differentiate between] ... phonological errors, stutter, and articulation errors'. One participant explained further, 'I generally try to categorise errors as being delayed or disordered based on the developmental norms, consistency vs. variability is NB in deciding if it's a phonological problem or a motor sequencing dyspraxia problem.' The comments made by participants indicate that they are using a range of widely recognised frameworks, even though they did not always explicitly label them.

\section{Intervention}

Eight participants (29\%) indicated that they have a 'core' therapy approach that they routinely use for children with speech difficulties. The remainder of the participants indicated that they do not have one specific approach. Some reasons given for not using a specific core approach were: 'Don't know of anything appropriate in Afrikaans'; 'I design treatment to suit each individual child'; 'Children present with 
individual speech production errors. Approach will thus depend on child's profile.' This illustrates that therapies are often combined and used in an eclectic manner, as SLTs consider an individual child and his/her presenting difficulties.

Table I shows how often different approaches are used by SLTs in their practice. The table divides these therapies into three categories (by collapsing responses to three levels), namely 'rarely/never', 'sometimes' and 'always/often'. It further divides therapies into two categories, namely popular therapies (those used always/often by more than $50 \%$ of participants) and less popular therapies (those used always/often by less than $50 \%$ ). This part of the analysis follows the Joffe and Pring (2008) paper.

Auditory discrimination, phonological awareness, parent-based programmes, articulation work/motor-skills training and core vocabulary are popular (used always/often by more than 50\%). Meaningful minimal-contrast pairs, cued articulation and the wholelanguage approach are among the popular therapies used overall by the participants in this survey; however they fell at the lower end of the popular category.

Participants were asked what factors influence their choice of approach. In most cases participants mentioned general criteria such as child's age $(n=10 ; 36 \%)$, frequency of therapy that can be offered $(n=10 ; 36 \%)$, and parent involvement $(n=8 ; 29 \%)$. More specific criteria related to the speech difficulty were also given, e.g. whether the child's errors are consistent or inconsistent $(n=8 ; 29 \%)$ and whether the child has delayed or disordered speech $(n=6 ; 22 \%)$. Many participants $(n=12 ; 43 \%)$ also commented on the importance of having family support.

The participants were asked to identify the factors that presented the greatest challenge when assessing and providing therapy to children with speech difficulties. The majority of participants commented that language presented a challenge when managing bilingual children, with one participant commenting on challenges related to bilingualism, saying '[difficult to make] differentiation between language delays versus bilingualism issues'. Other challenges included limited resources or lack of standardised tests, and participants commented: '[difficult to decide] which children to refer to speech therapy', 'inappropriate material' and 'inconsistent attendance due to social situations of family', 'lack of accountability of the Department of Education in providing support in schools for these children?.

Participants were asked about their awareness of the evidence base for intervention for children with speech disorders. Most of the participants strongly agreed/agreed that there is sufficient evidence to show that intervention for children with speech disorders is effective $(85 \%, n=24)$. The remaining participants either reported that they did not know $(4 \%, n=1)$, disagreed $(4 \%, n=1)$ or did not respond $(7 \%, n=2)$.
None of the participants referred to the evidence base when justifying their selection of therapy methods.

\section{Discussion}

Children with speech difficulties comprise a substantial proportion (40-70\%) of the caseloads of the majority of SLT participants in this study. In South Africa, most children presenting with speech difficulties will be bilingual or multilingual. The survey found that the majority of the SLT participants working with children with speech difficulties in the Western Cape see children who are multilingual speakers of the main languages, Afrikaans, isiXhosa and English. However, children who speak isiXhosa and have speech difficulties are at a considerable disadvantage because there are few therapists in the region who can speak isiXhosa ( $n=1 ; 4 \%$ in the present study). Furthermore, of the SLTs available to work with isiXhosa children in the region, only $40 \%$ are able to make use of interpreters or problem solve in creative ways so that these children can be assessed and managed in their first language. It is important to note that results from this survey show that a considerable proportion of SLTs are only able to offer therapy in English (or Afrikaans) - even when working with children who have these as a second or third language. There is a fundamental challenge here: ethical guidelines suggest a child should not be denied intervention because of a language mismatch with the clinician, but SLTs may not be competent to offer therapy in all languages. A study by Jordaan and Yelland (2003) attempted to determine how South African SLTs provide language intervention for multilingual language-impaired children. Similar to the present study, the results indicated that the majority of SLTs are providing language therapy to multilingual children in the child's second language only - typically English. Jordaan and Yelland (2003) attributed this to parental insistence and a lack of another common language between the SLT and child. This finding confirms that there is a lack of African-language-practising SLTs in South Africa, and many children are therefore not being treated for speech and/or language disorders in their mother tongue.

There are few assessments, therapy materials and a lack of published literature available that deal with languages other than English, and the challenges facing SLTs are considerable. It is not surprising that most participants do not feel confident in selecting assessment and therapy approaches when managing children with speech difficulties, especially when the children are multilingual. Many of the participants who rated themselves as not very confident/not confident at all were very experienced (having worked for more than 10 years). This may be related to differences in training of SLTs, where the training of those who graduated before 1994 was focused mainly on treating children in English or Afrikaans. Newly graduated SLTs are now exposed to different teaching methods to overcome language as a barrier to assessment and intervention. SLTs should be constantly improving their language skills so that they can practise cross-linguistically, and should make every attempt to problem solve around such issues.

Table I. Percentage of participants $(n=28)$ using individual therapies

$\begin{array}{lcrr}\text { Category } & \text { Rarely/never } & \text { Sometimes } & \text { Always/often } \\ \text { Popular therapies } & & & 89 \\ \text { Auditory discrimination } & 0 & 11 & 79 \\ \text { Phonological awareness (Gillon, 2000) } & 0 & 21 & 74 \\ \text { Parent-based programmes } & 7 & 19 & 61 \\ \text { Articulation work/motor-skills training (Van Riper \& Emerick, 1984) } & 14 & 25 & 50 \\ \text { Core vocabulary (Dodd, 2005) } & 26 & 43 & 44 \\ \text { Meaningful minimal-contrast pairs (Weiner, 1981) } & 7 & 19 & 42 \\ \text { Cued articulation (Passey, 1990) } & 37 & 36 & 8 \\ \text { Whole language approach (Hoffman et al., 1990) } & 22 & 24 & 18 \\ \text { Less popular therapies } & 68 & 25 & 18 \\ \text { Metaphon (Howell \& Dean, 1991) } & 57 & 26 & 23 \\ \text { Suck, swallow, breathe synchrony (Oetter et al., 1993) } & 59 & 29 & 11 \\ \text { Maximal contrast therapy (Gierut, 1990) } & 54 & 19 & 22 \\ \text { Non-speech oro-motor work (Lancaster \& Pope, 1989) } & 58 & 31 & 32 \\ \text { Nuffield Centre Dyspraxia Programme (Williams \& Stephens, 2004) } & 58 & 32 & \\ \text { Cycles (Hodson \& Paden, 1991) } & 46 & & \\ \text { Auditory bombardment (Hodson \& Paden, 1991) } & & & \\ \end{array}$




\section{Assessment}

Of the SLTs surveyed, it was found that $89 \%(n=25)$ use informal assessment tools in addition to formal assessments. This differs from the findings by Joffe and Pring (2008) from their study of UK-based therapists, and shows the contextual differences between the two settings. In the Western Cape the main languages are Afrikaans, isiXhosa and (South African) English and there are few, if any, assessments that have been specifically developed and validated for these populations. Western Cape SLTs therefore rely largely on informal assessments when evaluating children's speech. They make adaptations to formal assessments, as well as using other informal assessments of their own design. More than $50 \%$ of therapists indicated that they make adaptations to formal tests to better suit the population, e.g. translating the assessment and using more contextually relevant pictures. These SLTs will often omit items or sections of formal assessments that are not appropriate for their clients, and will administer tests in non-standardised ways, e.g. repeating instructions or test items. Few SLTs specified how the results from such assessments are interpreted, but presumably the norms are then used as guidelines only, and the focus is on a qualitative description. This thoughtful engagement with assessment issues is to be commended, but raises two important issues. Firstly, since there are clearly many SLTs who have created/adapted assessments, it may be valuable to share these resources through a common forum which could advance the development of such assessments in a systematic manner and ensure that new SLTs or those setting up services would not have to 'reinvent the wheel'. Secondly, there is clearly a need for speech assessments that have been properly validated and normed on local populations, so that all clients can be accurately and reliably assessed. This is a need that must be addressed if SLTs are to appropriately serve all clients requiring services. In the current situation, many clients are not having their speech difficulties managed because they do not speak English as their first language. Those who are assessed will, for the most part, not be assessed using valid and reliable tools; this in turn creates difficulties in planning and evaluating intervention.

In terms of formal tests, the GFTA (1986) was the most popular assessment used. This is surprising for a number of reasons. Like many of the other tools used by South African SLTs it has been developed overseas and standardised on a different population. The GFTA elicits phonemes in only one specific phonetic context within a preselected word. It provides information about the child's phonetic inventory but does not involve phonological analysis or detail whether sound errors are developmentally appropriate. Like many other speech assessments it focuses primarily on single words and not connected speech. Research suggests that the discrepancy between single-word and connected speech phonology may be considerable, and is an important factor to take into account in intervention planning (Pascoe et al., 2006). The way in which the GFTA was used was not always fully detailed by respondents, but there were SLTs in this study who use the GFTA pictures as a starting point for eliciting single words, before going on to elicit a connected speech sample, and then using their own system of analysis to interpret the results.

The informal assessment tools used by SLTs in the Western Cape reflect the way in which SLTs think about speech disorders. The psycholinguistic approach was often implicitly followed. For example, sets of pictures were devised by participants or taken from formal assessments and used in confrontation naming tasks to access lexical representations. There was however a bias in both assessment and intervention towards output aspects of speech processing (naming, repetition) rather than tasks which focused primarily on auditory input or mispronunciation detection. Joffe and Pring (2008) found that SLTs had little enthusiasm for frameworks in which a more detailed assessment could be carried out, with many SLTs reporting their 'fear' of the psycholinguistic approach. However, in this study, this was not the case, as most of the SLTs make use of certain aspects of the psycholinguistic approach. Stackhouse and Wells (1997) note that all materials can be used psycholinguistically and that it is an approach carried in the head of the user rather than in a specific box of equipment.
In recent years there has been an increase in awareness around cultural appropriateness of assessment tools in South Africa and many of the SLTs surveyed are making necessary adaptations to overcome related issues; however, more information in this regard is needed in order to make the assessment of speech difficulties in children more effective. Most respondents noted that a speech assessment that enabled them to assess all three of the main Western Cape languages would be useful. They envisaged a test with modern, colourful pictures appropriate for all children living in the region. In addition it would be valuable to know more about accepted variations of speech sound production in the Western Cape, have norms for speech development in the region, e.g. a list of phonological processes in each of the languages, and have clinical judgement frameworks to assist in decision making regarding disordered or delayed profiles. While the importance of formal assessment is emphasised here, SLTs should also be cautious not to rely solely on such tools. Clearly a thorough assessment will entail integration of formal and informal assessment tasks, clinical judgement and input from the child and significant others.

\section{Intervention}

A wide range of approaches and techniques are available for managing speech difficulties in children. However, there is limited literature addressing the issue of how to choose between the treatments options (Dodd, 2005) and the evidence base regarding the effectiveness of the approaches is still in its infancy.

Like those in the study by Joffe and Pring (2008), SLTs surveyed in our sample use a wide variety of therapies, often in an eclectic way. Few therapists adhere to one core approach when treating children with speech difficulties. This seems entirely appropriate, as authors such as Bowen (2009) note that management of speech difficulties should be adjusted to suit the diversity of each client, and therapy should consist of a combination of approaches most suitably tailored for each individual. Evidence-based practice is about choosing the most appropriate intervention for a particular client, based on best available evidence as well as therapist experience and client needs.

In the study by Joffe and Pring (2008) the most popular therapies used were auditory discrimination, phonological awareness and parent involvement. The results of the present study follow closely in that $89 \%$ of participants used auditory discrimination, 79\% used phonological awareness and $74 \%$ used parent involvement. The popularity of auditory work in both studies warrants further investigation. Children with speech difficulties may have strong auditory processing skills even in the context of severe output difficulties, therefore Joffe and Pring (2008) concluded that in these cases, work on auditory discrimination may be unnecessary. However, input-based therapy may be a useful way of accessing the phonological system of children with persisting speech difficulties who are unwilling to produce speech, perhaps as a result of years of therapy focused on their speech output (Pascoe et al., 2006). In our questionnaire 'auditory discrimination' was included as a general approach to therapy, rather than referring to a specific programme of therapy. However, auditory discrimination activities could be carried out at a variety of levels (e.g. single sounds, words, sentences) and in a variety of ways (e.g. as preparation for a minimal-pair approach to therapy that focuses on output, or as the main intervention in its own right). The questionnaire did not allow SLTs to indicate how they incorporate auditory activities into their interventions. Another more specific auditory-based approach, that of auditory bombardment (Hodson \& Paden, 1991), proved considerably less popular.

Phonological awareness approaches were popular in both studies. In our study (as for the Joffe and Pring (2008) paper) we included phonological awareness approaches such as those described by Gillon (2000) which focus on using phonological awareness activities as a way to access and improve phonological representations. There is evidence that such approaches are effective in bringing about change in children's speech, phonological awareness and early literacy (Bowen, 2009; Gillon, 2000). Again, the way in which therapists in our study carry out 
phonological awareness activities was not explored, and the focus of the interventions (and rationale underpinning them) may in fact vary widely, e.g. phonological awareness may include aspects of input and/ or output processing at a variety of levels.

Parent involvement has long been described as key to effective intervention (Bowen, 2009) but might be facilitated in a variety of ways. In some cases 'home programmes' are used as the sole form of intervention; in other instances parents play a key role in therapy (e.g. the PACT approach (Bowen \& Cupples, 2006) and in core vocabulary approaches (Dodd, 2005). In some instances parents are involved to a lesser extent, often as a result of resource constraints. In this study most of the SLTs adopt a parent-based approach to therapy which follows the Joffe and Pring study where $76.5 \%$ of SLTs included parental involvement in their intervention programmes, but again this does not mean that all SLTs involve parents in the same way.

The effectiveness of many of the approaches used by the SLTs in our study has been demonstrated, although often in small-scale or inconsistent ways (Williams et al., 2010). In describing the decision-making process, SLTs referred to multiple factors such as a client's age, the nature of their speech difficulties and family support, but did not mention the available evidence base as a guiding factor in their selection. Joffe and Pring (2008) describe this as a gap between research and practice. In the present study, SLTs were asked about their awareness of the evidence base in the area of children's speech difficulties and the majority (85\%) agreed that there is sufficient evidence to show that intervention for children with speech disorders is effective. However, none of the participants referred to the evidence base when justifying their selection of therapy methods, suggesting that they are either not familiar with the recent evidence or that evidence-based practice may be a concept that SLTs find hard to apply to their own work - the research/practice gap that Joffe and Pring describe.

As for the assessment section of the questionnaire, most of the respondents described challenges in terms of intervention that focused on working with bi/multilingual children and a lack of resources. SLTs also mentioned the social/financial difficulties that may make it hard for clients to attend appointments. This raises a bigger issue for the profession as a whole regarding service delivery that is relevant and not 'hard to reach' (Lees, 2002). Given that there are no SLTs currently employed in ordinary mainstream schools in the Western Cape, there are further challenges in accessing children who need intervention.

\section{Limitations of the study}

The study reports results from a small sample of local SLTs. The response rate was below the desirable response rate described in the literature (Maxwell \& Satake, 2006) and therefore findings cannot be generalised to the greater population. Furthermore the findings may be biased because the SLTs who did respond are more likely to have been interested in research, and interested in issues of linguistic diversity than those who had engaged less with the topic. However, findings do provide insight into the methods of assessment and intervention used by some SLTs in this region, and may serve as a start in exploring clinical practice of this group.

The use of questionnaires presents difficulties in the collection of data. In this study this included the low response rate, issues of validity and difficulties with some of the questions. For example, question 16 posed difficulties for many respondents as it consisted of two questions in one: 'Are you aware of or do you use any frameworks for categorising children with speech difficulties? Which features would help you to identify a child with a phonological delay; phonological disorder or dyspraxia?' Many of the participants only answered the second part of the question, thus making it difficult to draw conclusions about SLT's awareness of frameworks. A more comprehensive piloting of the questionnaire would have been helpful in detecting some of these difficulties, and increasing the validity of the instrument.
Carrying out face-to-face interviews may have increased the sample size and yielded more in-depth information; however, it may have been difficult because of SLTs' time constraints. Another possible alternative would be to conduct a similar study with a larger sample size (i.e. including SLTs in South Africa as a whole). The information obtained from a national survey may be more valuable than merely looking at the local context and would not require significantly more resources since most of the questionnaires were sent via electronic mail.

\section{Clinical implications}

Almost all the survey respondents described similar challenges in managing children with speech difficulties, linked mainly to a lack of appropriate resources and compounded by the fact that there is often a mismatch between clinician and client's languages. Clinicians in the Western Cape, and in South Africa as a whole, can constructively address these issues by sharing multi-language resources they may have developed with other clinicians and offering critique and support in the ongoing development of such materials to ensure that quality service delivery is maintained. It is exciting to know that there are isiXhosa screening tools in use, and it would be helpful to share these and develop them further. Where therapists are adapting assessments, they should think critically about the nature of these adaptations: What is really being assessed? Is this fair and appropriate given the client's language/s? How do the data collected inform therapy planning? Ongoing engagement with the literature on speech difficulties and bilingual speech and language development will ensure that a gap between research and practice does not develop. Clinicians should be mindful of the current state of the evidence base in this area. There is strong and clear evidence about which approaches work best with which children, but clinicians working in the South African context are well positioned to add to this evidence base and grow the knowledge in a local context. The calls made by SLTs in this study for lists of phonological processes in more languages and accepted variations of speech sounds produced in particular regions are basic tools that are urgently needed.

\section{Further research}

In South Africa there is a great need to develop new and appropriate assessment tools in languages other than English. Further, knowledge regarding the development of speech in the different languages is needed for SLTs as this could be used as a guideline for both assessment and intervention for children with speech difficulties in these languages. SLTs will not be able to achieve this in isolation. We should collaborate with each other at a national level, look internationally for lessons and guidelines in materials development and translation, and link with our academic colleagues working in disciplines such as linguistics and psychology. More evidence about speech difficulties in languages such as Afrikaans and isiXhosa is needed as most of the studies have been done with English-speaking children. There is also a need for intervention studies on the South African population in order to build an evidence base for the different approaches used.

\section{Conclusion}

This study found that the majority of SLTs working in the Western Cape assess and manage children with speech difficulties as a substantial part of their caseloads. Informal assessments and adapted versions of published tests are widely used. The SLTs surveyed draw on a wide range of intervention approaches and use them most often in combination. Most of the participants do not feel confident in selecting assessment and therapy approaches when managing children with speech difficulties, specifically when managing multilingual children. This was associated with challenges related to the language diversity of the children in this region, the lack of resources and the lack of assessments standardised on this population. There is a need for further research into typical speech development in the South African context, as well as development of resources tailored to the population.

\section{Acknowledgements}

Thanks to all the clinicians who participated in the study, and to Jenny Pahl for her helpful comments on an earlier draft. This research was funded by the National Research Foundation (South Africa). 


\section{References}

Anthony, A., Bogle, D., Ingram, T., \& McIsaac, W. (1971). The Edinburgh articulation test. Edinburgh: Livingstone.

Armstrong, S., \& Ainley, M. (1988). The South Tyneside assessment of phonology. Northumberland: STASS.

Bird, J, Bishop, D. V. M., \& Freeman, N. H. (1995). Phonological awareness and literacy development in children with expressive phonological impairments. Journal of Speech and Hearing Research 38, 446-462.

Bowen, C. (2009). Children's speech sound disorders. Oxford: Wiley-Blackwell.

Bowen, C., \& Cupples, L. (2006). PACT: Parents and children together in phonological therapy. Advances in Speech-Language Pathology, 8, 282-292.

Broomfield, J., \& Dodd, B. (2004). Children with speech and language disability: caseload characteristics. International Journal of Language and Communication Disorders, 39, 303 324.

Carter, J., Lees, J., Murira, G., Gona, J., Neville, B., \& Newton, C. (2004). Issues in the development of cross-cultural assessments in speech and language for children. International Journal of Language and Communication Disorders, 4, 385-401.

Dodd, B. (Ed.). (2005). Differential diagnosis and treatment of children with speech disorder (2nd ed.). London: Whurr Publishers.

Gardner, M. F. (1996). Test of auditory perceptual skills: Revised. Burlingame, CA: Psychological and Educational Publications.

Gierut, J. (1990). Differential learning of phonological oppositions. Journal of Speech and Hearing Research, 33, 540-549.

Gillon, G. T. (2000). The efficacy of phonological awareness intervention for children with spoken language impairment. Language, Speech and Hearing Services in Schools, 31, 126141.

Goldman, R., \& Fristoe, M. (1986). Goldman-Fristoe test of articulation. Circle Pines, MN AGS.

Goldstein, B. A., \& Fabiano, L. (2006). Assessment and intervention of children with phonological difficulties. Early intervention in depth. Rockville, MD: The American Speech and Hearing Association.

Grunwell, P. (1985). Phonological assessment of child speech. NFER-Nelson: Windsor.

Gxilishe, S. (2004). The acquisition of clicks by Xhosa speaking children. Per Linguam, 2, 1-12.

Hodson, B., \& Paden, E. (1991). Targetting intelligible speech: A phonological approach to remediation. San Diego, CA: College Hill Press.

Hoffman, P., Norris, J., \& Monjure, J. (1990). Comparison of process targeting and whole language treatments for phonologically delayed preschool children. Language, Speech and Hearing Services in Schools, 21, 102-109.
Howell, J., \& Dean, E. (1991). Treating phonological disorders in children: Metaphon - theory to practice. London: Whurr Publishers.

Joffe, V., \& Pring, T. (2008). Children with phonological problems: a survey of clinical practice. International Journal of Language \& Communication Disorders, 43, 154-165.

Jordaan, H., \& Yelland, A. (2003). Intervention with multilingual language impaired children by South African SLTs. Journal of Multilingual Communication Disorders, 1, 13-33.

Lancaster, G., \& Pope, L. (1989). Working with children's phonology. Bicester: Winslow.

Lees, J. (2002) Crossing the cultural divide. Bulletin of the Royal College of Speech and Language Therapists, October, 11-12.

Maxwell, D. L., \& Satake, E. (2006). Research and statistical methods in communication sciences \& disorders (1st ed.). Boston: Thomson Delmar Learning.

Nash, P., Stengelhofen, J., \& Brown, J. (2002). Improving children's communication: Managing persistent communication difficulties. Chichester: Wiley.

Oetter, P., Richter, E., \& Frick, S. (1993). MORE: Integrating the mouth with sensory and postural functions. Hugo, MN: PDP.

Pascoe, M., Stackhouse, J., \& Wells, B. (2006). Persisting speech difficulties in children: Children's speech and literacy difficulties 3. Chichester: Wiley.

Passey, J. (1990). Cued articulation. Melbourne: ACER.

Reilly, S., Douglas, J., \& Oates, J. (2004). Evidence-based practice in speech pathology London: Whurr Publishers.

Robertson, C., \& Salter, W. (1995). The phonological awareness kit. East Moline, IL Linguisystems.

Ruscello, D. M. (2008). Treating articulation \& phonology disorders in children. St Louis Mosby Elsevier.

Stackhouse, J., \& Wells, B. (1997). Children's speech and literacy difficulties: A psycholinguistic framework. London: Whurr Publishers.

Stackhouse, J., Pascoe, M., \& Gardner, H. (2006) Intervention for a child with persisting speech and literacy difficulties: A psycholinguistic approach. International Journal of Speech-Language Pathology, 3, 231-244.

Van Riper, R., \& Emerick, L. (1984). Speech correction: An introduction to speech pathology and audiology. Englewood Cliffs, NJ: Prentice-Hall.

Weiner, F. (1981). Treatment of phonological disability using the method of meaningful minimal contrast: Two case studies. Journal of Speech and Hearing Disorders, 46, 97-103.

Williams, A. L., McLeod, S., \& McCauley, R. (2010). Interventions for speech sound disorders in children. Baltimore, MD: Paul Brookes.

Williams, P., \& Stephens, H. (Eds.). (2004). Nuffield Centre Dyspraxia Programme. Windsor, UK: The Miracle Factory.

\section{Appendix A}

\section{QUESTIONNAIRE}

\section{A. General Questions:}

1. Do you work with children? (Please circle)

YES / NO (If no, please proceed to question 22)

2. How long have you been a Speech-Language therapist? (Please circle)

Less than 1 year / Between 1 and 3 years / Between 4 and 6 years / Between 7 and 10 years / More than 10 years

3. In which setting/settings do you work? (Please circle)

Private practice / State hospital / Private hospital / State school / Private school / University / Non-governmental organisation (NGO) / Other (Please specify)

4. What language/s do you speak? (Please circle) English / Afrikaans / Xhosa / Other (Please specify)

5. Please describe the main client group that comprise your individual case load. (Please complete using the following example) Example: $10 \%$ children with phonological problems $30 \%$ children with fluency difficulties $50 \%$ feeding difficulties $10 \%$ autistic speech

6. What percentage of your current (individual) caseload is taken up with clients with speech difficulties? (NOTE: by speech difficulties we mean children who have difficulties in producing clear, fluent, well-articulated or intelligible speech) (Please circle) Less than 10\% / Between 10\% and 39\% / Between 40\% and 70\% / Greater than 70\%

7. Please indicate whether you work with children that speak the following languages: (Please circle) English / Afrikaans / Xhosa / Other (Please specify)

8. Focusing on children's main language (L1), how many children that are part of your caseload have speech difficulties in the different languages? English L1__ $\%$ / Afrikaans L1__ $\%$ / Xhosa L1__ $\%$ / Other L1___ \% 
9. How confident do you feel assessing and providing therapy to a child that speaks another language to your own? (Please circle most appropriate response)

Very confident / Confident / Neutral / Not very confident / Not confident at all

10. What language(s) do you use when assessing and providing therapy? (Please circle)

English / Afrikaans / Xhosa / The child's home language

B. Assessment:

The following questions are based on your assessment methods for evaluating a child's speech. (i.e. focusing primarily on articulation and phonology)

$\underline{\text { Standardised assessments }}$

11. Do you use any standardised assessments? (Please select, by circling an appropriate answer)

If no, why not?

If yes, what assessments do you use? (Please list)

12. Do you make any adaptations to these standardised assessments?

If yes, please explain why and how (please provide an example if possible):

Informal assessments

13. Do you use informal assessments?

Please describe in further detail (and see questions below).

Test items:

13a. Do you use: (Please circle)

pictures / objects / both / none

13b. Please describe your test items:

Pictures:

$\begin{array}{lll}\text { Colour } & \text { or } & \text { Black and white } \\ \text { Big } & \text { or } & \text { Small } \\ \text { Line drawings } & \text { or } & \text { 2-dimensional } \\ \text { Single picture presentation } & \text { or } & \text { multiple picture presentation }\end{array}$

or multiple picture presentation

\begin{tabular}{lll}
\hline Objects & & \\
Big & or & Small \\
Single object presentation & or & multiple object presentation
\end{tabular}

If you are able to include sample words from the informal assessment please do so.

14. Would an assessment used in all three predominantly used languages (English, Afrikaans, and Xhosa) be helpful to you in your current practice? Please specify yes/ no and comment as appropriate.

15. What additional materials would you find helpful in assessing a child's speech?

16. Are you aware of or do you use any frameworks for categorizing / diagnosing/ describing children with speech difficulties? Which features would help you to identify a child with a phonological delay; phonological disorder or dyspraxia?

\section{Intervention/Therapy:}

17. Do you have a core treatment approach or package that you routinely use for children with speech difficulties? Yes - Which one and why?

No - Why? 
18. Below is a list of common treatment approaches used to treat speech disorders. By circling, please rate how frequently you use each approach in your therapy using the scale below: 5 Always / 4 Often / 3 Sometimes / 2 Rarely / 1 Never

Meaningful minimal contrast therapy

(Weiner, 1981)

Maximal contrast therapy (Gierut, 1990)

Articulation work/motor skills training

(Van Riper \& Emerick, 1984)

Suck-swallow-breathe synchrony

(Oetter, Richter \& Frick, 1993)

Non-speech oro-motor work

(Lancaster \& Pope, 1989)

Nuffield Centre Dyspraxia Programme

(Williams \& Stephens, 2004)

Core vocabulary (Dodd, 2005)

Whole-language approach

(Hoffman, Norris \& Monjure, 1990)

Auditory bombardment

(Hodson \& Paden, 1991)

Metaphon (Howell \& Dean, 1991)

Phonological awareness (Gillon, 2000)

Cued articulation (Passey, 1990)

Cycles approach (Hodson \& Paden, 1991)

Parent-based work

$\begin{array}{lllll}5 & 4 & 3 & 2 & 1\end{array}$

Other:

19. Of these approaches, which do you find to be most effective?

20. Do you incorporate home programmes (Please circle)?

Always / Often / Sometimes / Rarely / Never

21. What factors do you consider when deciding on an appropriate treatment programme in the remediation of speech difficulties?

22. Being in a South African context, what are your greatest challenges faced with regard to assessment and therapy with children with speech difficulties? Language

Culture

Resources

Lack of standardised tests

Other, elaborate:

23. Please state whether you agree or disagree with this statement:

'I feel there is sufficient evidence to show that intervention for children with speech disorder is effective'

Strongly agree / Agree / Neither agree nor disagree / Disagree / Strongly disagree 